\title{
EDITORIAL
}

\section{A special review collection on autophagy}

Cell Research (2020) 30:553; https://doi.org/10.1038/s41422-020-0361-2

The popular perception is that awarding a Nobel prize to scientists in a certain field marks the maturation or even the decline of that field. Many have argued that few fundamental questions remain to be answered afterwards. In reality, the opposite is more likely to be true. The award of a Nobel prize to Dr. Ohsumi in 2016 sparked interest in autophagy from researchers across a wide range of research directions, and in the past few years, we have witnessed the robust growth of the autophagy field. Difficult but fundamental questions are being addressed with increasing clarity and, more importantly, new questions are arising from unexpected directions. Guangchao Chen and I organized a review collection on Autophagy published in Cell Discovery, presented recent advances in our understanding of autophagy with a focus on these two aspects of autophagy research.

The mechanism underlying autophagosome biogenesis is a fundamental question which arose in the early days of work on autophagy. In the past, the study of autophagosome biogenesis was largely protein centered; recently, with more understanding of how ATG proteins interact with membranes and membrane lipids, and with membrane lipids emerging as important regulators of autophagosomes, the mystery of autophagosome biogenesis is gradually being revealed. Drs. Nishimura and Tooze summarize the recent progress on this important front. For a long time, lysosomes were considered as the boring end of autophagy, a place where the autophagy cargos are dumped and degraded. Recently, a more complicated picture has emerged: lysosomes can be reformed and activated during autophagy, they can actively regulate autophagy, and damaged lysosomes can be targeted for degradation by autophagy. These important advances are summarized by Drs. Yim and Mizushima. ${ }^{2}$ The discovery by Dr. Ohsumi of Atg8 lipidation by two ubiquitin-like systems was the climax of earlier efforts to understand the biochemical nature of ATG proteins. ${ }^{3}$ An increasingly clear picture of the mechanism and function of these two ubiquitin-like systems on autophagy is emerging from recent work. More importantly, surprising facts about Atg8 lipidation keep coming out, including its role in autophagy-independent processes, and its apparently non- essential roles in autophagy in at least some mammalian cell lines. These advances are summarized by Drs. Martens and Fracchiolla. ${ }^{4}$ In the past few years, tremendous progress has been made in understanding the roles of autophagy in various diseases. These achievements chiefly come from two research strategies: on one hand, known autophagy genes are studied in various disease models to uncover their roles in disease pathology; on the other hand, mutations in autophagy genes are found to be associated with various diseases. We cover this important topic with two reviews. Drs. Kawabata and Yoshimori ${ }^{5}$ provide a comprehensive review summarizing the molecular regulation of autophagosome biogenesis and the contributions of its malfunction to various diseases. Dr. Rubinztein and his co-authors ${ }^{6}$ provide a review focusing on mutations that have been identified in autophagyrelated genes and their possible roles in neurodegenerative diseases.

We hope these excellent reviews will not only convey the new progress to experts in the field, but also provide new-comers with the basic framework of autophagy and help them understand the trends and possible future directions of this fascinating field.

\footnotetext{
$\mathrm{Li} \mathrm{Yu}$

${ }^{1}$ The State Key Laboratory of Membrane Biology, Tsinghua University-Peking University Joint Centre for Life Sciences, Beijing Frontier Research Center for Biological Structure, School of Life Sciences, Beijing 100084, China Correspondence: Li Yu (liyulab@mail.tsinghua.edu.cn)
}

\section{REFERENCES}

1. Nishimura, T. \& Tooze, S. A. Cell Discov, 6, 32 (2020).

2. Yim, W. W. \& Mizushima, N. Cell Discov. 6, 6 (2020).

3. Tsukada, M. \& Ohsumi, Y. FEBS Lett. 333, 169-174 (1993).

4. Martens, S. \& Fracchiolla, D. Cell Discov. 6, 23 (2020).

5. Kawabata, T. \& Yoshimori, T. Cell Discov. 6, 33 (2020).

6. Stamatakou, E. et al. Cell Discov. 6, 24 (2020). 